\title{
Deubiquitinating enzyme 4 facilitates chemoresistance in glioblastoma by inhibiting P53 activity
}

\author{
NA QIN $^{1 *}$, FENGLI HAN $^{2 *}$, LI LI $^{1}$, YINGWEI GE ${ }^{1}$, WEI LIN ${ }^{3}$, JIANG WANG $^{3}$, \\ $\mathrm{LIN} \mathrm{WU}^{4}$, GANG ZHAO ${ }^{1}$, YANCHUN DENG ${ }^{1}$ and JIAN ZHANG ${ }^{4}$ \\ ${ }^{1}$ Department of Neurology, Xijing Hospital, The Fourth Military Medical University; ${ }^{2}$ Department of Neurology, \\ The Fourth People's Hospital of Shaanxi; ${ }^{3}$ Department of Neurosurgery, Xijing Hospital, \\ The Fourth Military Medical University; ${ }^{4}$ Department of Biochemistry and Molecular Biology, \\ The Fourth Military Medical University, Xi'an, Shaanxi 710032, P.R. China
}

Received February 20, 2017; Accepted August 10, 2018

DOI: $10.3892 / \mathrm{ol} .2018 .9654$

\begin{abstract}
Glioblastoma is a malignant primary brain tumor with poor prognosis with a median survival of only 12-15 months. The high mortality rate of this disease is mainly due to the chemoresistance resulting from various reasons. Ubiquitin-specific protease 4 (USP4) has recently been found to be elevated in various types of cancer through regulating P53 activity. However, whether USP4 is responsible for chemoresistance in glioblastoma is not clear. In the present study, the expression of USP4 in glioblastoma tissues and cell lines, as well as its association with temozolomide (TMZ) chemoresistance was analyzed. The results demonstrated that USP4 was significantly upregulated in glioblastoma tissues and cell lines at the mRNA and protein levels. Notably, USP4 knockdown alone did not affect glioblastoma cell viability; however, when USP4 knockdown cells were treated with TMZ, the cell viability was decreased significantly. In addition, the results revealed that cleaved poly(ADP-ribose) polymerase level increased when USP4 was knocked down in glioblastoma cells treated with TMZ. It was also observed that P53 was increased in U251 and U87 cells with USP4 knockdown. Following treatment with a P53 specific inhibitor, the results suggested that USP4 mediated chemoresistance through inhibiting apoptosis in a P53-dependent manner. In conclusion, the data revealed
\end{abstract}

Correspondence to: Dr Yanchun Deng, Department of Neurology, Xijing Hospital, The Fourth Military Medical University, 15 Chang Le West Road, Xi'an, Shaanxi 710032, P.R. China

E-mail: yanchund@fmmu.edu.cn

Dr Jian Zhang, Department of Biochemistry and Molecular Biology, The Fourth Military Medical University, 15 Chang Le West Road, Xi'an, Shaanxi 710032, P.R. China

E-mail: biozhangj@163.com

*Contributed equally

Key words: ubiquitin-specific protease 4, P53, glioblastoma, chemoresistance, RNA interference the critical role of USP4 in TMZ resistance in glioblastoma and provided new insight for future drug development for the treatment of this disease.

\section{Introduction}

Glioblastoma is one of the most common primary brain tumors in adults, and glioblastoma alone accounts for $\sim 70 \%$ of high-grade gliomas (1). Despite the advances in chemotherapy, radiotherapy, surgical resection and most recently immunotherapy, the prognosis of patients with glioblastoma remains poor, with a median survival of 12-15 months (2-6). Temozolomide (TMZ) is the most common drug in glioblastoma chemotherapy and is used throughout the whole treatment of glioblastoma (7). However, the efficacy of TMZ is limited partly due to the high activity of DNA repair in tumor cells, which reduces the effect of this alkylating agent and leads to a resistant phenotype. Multiple theories have been developed to explain the TMZ resistance in glioblastoma $(8,9)$, including DNA repair mechanism (10), overexpression of epidermal growth factor receptor and galectin-1 $(11,12)$, malfunction of p53 (13), Murine double minute 2 (14), and phosphatase and tensin homolog (15), as well as involvement of miRNAs $(16,17)$. The P53 pathway is inactivated in almost $50 \%$ of human tumors (18). Malfunction of P53 generally leads to a poorer prognosis for cancer patients, and accumulating studies have demonstrated that chemoresistance is associated with P53 inactivation (18-20). Since TMZ functions by inserting alkyl groups into DNA to cause DNA damage and inhibit cell division, mutation of P53 inhibits its regulative role in DNA replication and repair, thus enhances the resistance of glioblastoma cells to TMZ $(21,22)$. Recently, BACH1 has been found to promote TMZ resistance in glioblastoma through antagonizing the function of p53 (23). Thus, it is critical to inspect P53 activity in glioblastoma cells with varying genetic background and to adjust the therapeutic plan according to specific conditions.

Ubiquitination is a critical regulatory event in cancer, particularly ubiquitination and deubiquitination of the proteins that affects p53 pathway activity. Deubiquitinating enzymes mediate the removal of ubiquitin and are divided into four 
subclasses based on their Ub-protease domains, including ubiquitin-specific proteases (USPs), ubiquitin C-terminal hydrolases, Otubain proteases and Machado-Joseph disease proteases (24). USPs have been reported to serve key roles in the progression of glioblastoma. For instance, USP15 amplification confers poor prognosis in individuals with glioblastoma through transforming growth factor- $\beta$ (TGF- $\beta$ ) signaling, while USP13 maintains glioblastoma stem cells by antagonizing Myc ubiquitination (25). Furthermore, USP7 was identified as a stabilizer of P53 by directly binding to P53 (26), and USP2 was found to facilitate the p53-mediated intrinsic apoptotic pathway in glioblastoma (27).

USP4 is a negative regulator of P53 by stabilizing ARF-BP1 and HDAC2, and has been found to be overexpressed in several types of human cancer $(28,29)$. In breast cancer, USP4 crosslinks protein kinase B and TGF- $\beta$ pathway to promote cancer cell migration (30). USP4 has also been demonstrated to control the potential of brain metastasis in patients with lung adenocarcinoma (31). Recently, increased USP4 levels were reported following intracerebral hemorrhage in adult rats, and this enzyme participated in neuronal apoptosis (32), indicating the critical role of USP4 in neuronal cells and apoptosis. However, the role of USP4 in glioblastoma is not currently clear, particularly when TMZ treatment is involved.

In the present study, the aim was to elucidate the role of USP4 in glioblastoma. The expression level of USP4 in glioblastoma tissues and cell lines was examined, while the chemoresistance of TMZ upon USP4 knockdown was tested. The study also inspected the role of P53 in USP4-mediated TMZ resistance, and the use of a specific inhibitor of P53 revealed the cancer promoting role of USP4 via the regulation of P53 activity.

\section{Materials and methods}

Cell culture. Human glioblastoma U251 and U87 cells were obtained from the American Type Culture Collection (Manassas, VA, USA). Primary human astrocytes were purchased from Procell Life Science \& Technology Co., Ltd (Wuhan, China). Cells were maintained in Dulbecco's modified Eagle's medium supplemented with $10 \%$ fetal bovine serum (Invitrogen; Thermo Fisher Scientific, Inc., Waltham, MA, USA) and 1\% penicillin/streptomycin (Invitrogen; Thermo Fisher Scientific, Inc.) at $37^{\circ} \mathrm{C}$ in a humidified atmosphere with $5 \% \mathrm{CO}_{2}$. All $\mathrm{TMZ}$ treatments were performed with $100 \mu \mathrm{M}$ TMZ (T257; Sigma-Aldrich; Merck KGaA, Darmstadt, Germany) for $24 \mathrm{~h}$, at $37^{\circ} \mathrm{C}$. The groups were divided as follow: Scramble (cells transfected with scramble siRNA), siRNA-1 (cells transfected with siRNA-1 targeting USP4) and siRNA-2 (cells transfected with siRNA-2 targeting USP4). TMZ+ indicates cells treated with $100 \mu \mathrm{M}$ TMZ while PFT+ indicates cells treated with $10 \mu \mathrm{M}$ PFT. PFT treatment were performed as previously described (33), with $10 \mu \mathrm{M}$ PFT (Tocris Bioscience, Bristol, UK) for $24 \mathrm{~h}$ at $37^{\circ} \mathrm{C}$. The cell lines were transfected with $50 \mathrm{nM}$ scramble small interfering RNA (siRNA) or each of the two siRNAs designed for USP4 [siRNA-1: 5'-GGCUCUGGAACA AAUACAU-3' and siRNA-2: 5'-GGUCGCAGAUGUGUAUAA U-3']. All transfections were performed using Lipofectamine ${ }^{\circledR}$ 3000 (Invitrogen; Thermo Fisher Scientific, Inc.), according to the manufacturer's protocol. After $24 \mathrm{~h}$ of siRNA transfection, cells were treated with TMZ with or without $10 \mu \mathrm{M}$ PFT for $24 \mathrm{~h}$ at $37^{\circ} \mathrm{C}$ prior to further analysis.
Human glioblastoma samples. Human glioblastoma tissue samples and their adjacent controls $(n=4)$ were obtained from patients who underwent surgical resection at the Department of Neurology at Xijing Hospital of the Fourth Military Medical University (Xi'an, China). These samples were confirmed as glioblastoma by pathological diagnosis (34). The present study was approved by the Ethics Committee of the Fourth Military Medical University. Written informed consent was obtained from all the patients.

USP4 small interfering RNA (siRNA) transfection in U251 and U87 cells. USP4 siRNA was designed and synthesized by Shanghai GenePharma Co., Ltd. (Shanghai, China), according to the sequence information of the USP4 gene (GenBank accession no. NM_001251877). The two siRNA sequences used in the study were as follows: (siRNA-1), 5'-GGCUCU GGAACAAAUACAU-3'; and siRNA-2, 5'-GGUCGCAGA UGUGUAUAAU-3'. A siRNA with a scrambled sequence (5'-UUCUCCGAACGUGUCACGU-3') was used as the negative control. U251 and U87 cells were seeded in 60-mm dishes at a density of $5 \times 10^{5}$ cells/dish and incubated overnight at $37^{\circ} \mathrm{C}$ in a humidified atmosphere containing $5 \% \mathrm{CO}_{2}$. Subsequently, cells were transfected with siRNAs using Lipofectamine ${ }^{\circledR}$ 3000 (Thermo Fisher Scientific, Inc.) according to the manufacturer's protocol. After $24 \mathrm{~h}$, cell lysates were collected for use in subsequent experiments.

Western blotting. U251 and U87 cells were washed with PBS twice and lysed in radioimmunoprecipitation assay lysis buffer (Thermo Fisher Scientific, Inc.). Proteins were quantified with a Pierce BCA Protein Assay kit (Thermo Fisher Scientific, Inc., Waltham, MA, USA) and $10 \mu \mathrm{g}$ protein for each sample was separated by $10 \%$ SDS-polyacrylamide gel electrophoresis and then transferred onto a nitrocellulose membrane (EMD Millipore, Billerica, MA, USA). The membrane was blocked at room temperature with $5 \%$ milk in Tris-buffered saline/Tween 20 (TBST; containing $20 \mathrm{mM}$ Tris- $\mathrm{HCl}, 150 \mathrm{mM} \mathrm{NaCl}$ and $0.1 \%$ Tween 20, $\mathrm{pH} 7.5$ ) for at least $1 \mathrm{~h}$ and then incubated with each primary antibody overnight at $4^{\circ} \mathrm{C}$. Primary antibodies against USP4 (ab38510; 1:500), p53 (ab131442; 1:1,000), poly(ADP-ribose) polymerase (PARP; ab32064; 1:1,000) were used, which were purchased from Abcam (Cambridge, MA, USA). Equal loading of samples was verified by immunoblotting for GAPDH (ab128915; 1:10,000; Abcam). Subsequently, the membrane was washed with TBST buffer for at least five times for $5 \mathrm{~min}$ each and then incubated with goat anti-rabbit secondary antibody (sc-2004, 1:5,000; Santa Cruz Biotechnology, Inc., Dallas, TX, USA) for $1 \mathrm{~h}$ at room temperature. The protein bands were visualized using a Pierce ${ }^{\mathrm{TM}}$ ECL Western Blotting Substrate (Thermo Fisher Scientific, Inc.).

Reverse transcription-quantitative polymerase chain reaction (RT-qPCR). Total RNA was extracted from U251 and U87 cells using TRIzol ${ }^{\circledR}$ reagent (Invitrogen; Thermo Fisher Scientific, Inc.), according to manufacturer's protocol. For each sample, 500 ng total RNA was reverse-transcribed using a Promega Reverse Transcription System (Promega Corporation, Madison, WI, USA), and the resulting complementary DNA was diluted 40 times. qPCR was then performed using a QuantiTect SYBR ${ }^{\circledR}$ Green PCR kit (204141; Qiagen China 
Co., Ltd., Shanghai, China) in 96-well optical reaction plates and the ABI StepOne Plus Real-Time PCR System (Applied Biosystems; Thermo Fisher Scientific, Inc.). The PCR reaction cycle was $95^{\circ} \mathrm{C}$ for $3 \mathrm{~min}$, followed by 40 cycles of denaturation at $95^{\circ} \mathrm{C}$ for $15 \mathrm{sec}$ and an annealing/elongation step at $60^{\circ} \mathrm{C}$ for $30 \mathrm{sec}$. The following primer sequences were used: Human GAPDH, 5'-GAAGATGGTGATGGGATTTC-3' (forward) and 5'-GAAGGTGAAGGTCGGAGTC-3' (reverse); human USP4, 5'-CCTGGGCTCTGTGGACTTG-3' (forward) and 5'-TGTTGATTTCGGCTTCATACTC-3' (reverse). USP4 transcription level was normalized to GAPDH as reference gene. Relative expressions values for are presented as fold-increase in relation to control. The actual values were calculated using the $2^{-\Delta \Delta \mathrm{Cq}}$ method (35).

MTT assay. U251/U87 cells transfected with control siRNA or USP4 siRNA were treated with $100 \mu \mathrm{M} \mathrm{TMZ}$ for $24 \mathrm{~h}$ at $37^{\circ} \mathrm{C}$. TMZ-treated and untreated cells were plated at a density of $1 \times 10^{4}$ cells/well in a 48 -well plate. Viable cells were then stained with $0.25 \mathrm{mg} / \mathrm{ml}$ MTT [also known as 3-(4,5-dimethylthiazol-2-yl)-2,5-diphenyltetrazolium bromide] for $1 \mathrm{~h}$. The media were then removed, and the formazan crystals produced were dissolved by the addition of dimethyl sulfoxide. Cell viability was determined according to the absorbance at $540 \mathrm{~nm}$.

Flow cytometry. Wild-type U251/U87 cells and U251/U87 cells transfected with control or USP4 siRNA were treated with $100 \mu \mathrm{M}$ TMZ or $10 \mu \mathrm{M}$ PFT for $24 \mathrm{~h}$. The TMZ-treated and untreated cells were seeded into 6 -well plates at a density of $1 \times 10^{6}$ cells/well for $24 \mathrm{~h}$. Subsequently, the cells were collected and stained with FITC Annexin V Apoptosis Detection Kit I (cat. no. 556547; BD Biosciences, San Jose, CA, USA) according to the manufacturer's protocol, and then analyzed by flow cytometry (FACSCalibur; BD Biosciences).

Immunohistochemical analysis. Tissues were fixed with $10 \%$ formalin for $2 \mathrm{~h}$ at room temperature, embedded in paraffin and then sectioned into 5-mm slides for USP4 expression analysis. The specimens were blocked for $1 \mathrm{~h}$ at room temperature with 10\% sheep serum (Beyotime Institute of Biotechnology, Haimen, China). The primary antibody was anti-USP4 rabbit polyclonal antibody (ab3850; Abcam; 1:100 dilution) at $4^{\circ} \mathrm{C}$ overnight, and the secondary antibody was goat anti-rabbit horseradish peroxidase-conjugated antibody (ab6721; Abcam; 1:1,000) at room temperature for $1 \mathrm{~h}$. The detailed IHC procedure was described previously (36).

Statistical analysis. Data are presented as the mean \pm standard error of at least three separate experiments. To assess significant differences between two groups, Student's t-test was performed. Statistical calculations were performed using IBM SPSS software (version 20.0; IBM Corp., Armonk, NY, USA). P-values of $<0.05$ were considered to denote differences that were statistically significant.

\section{Results}

Increased USP4 expression in glioblastoma tissues and cell lines. To investigate the role that USP4 plays in glioblastoma, the mRNA and protein expression levels of USP4 were first examined in the glioblastoma cell lines U251 and U87. The RT-qPCR results demonstrated that UPS4 mRNA expression was significantly increased in U251 and U87 cell lines by $\sim 3$ folds, as compared with primary human astrocytes as negative control cells (Fig. 1A). The protein level of USP4 was also evidently increased in the two cell lines (Fig. 1B). Next, the expression of USP4 in glioblastoma tissues was tested by immunohistochemical staining. The results revealed that USP4 was highly expressed in cancer tissues compared with control tissues (Fig. 1C).

Downregulation of USP4 ameliorates chemoresistance of U251 and U87 cells to TMZ. In order to further explore the function of the elevated USP4 in glioblastoma, USP4 was knocked down by two different siRNA sequences in U251 and U87 glioblastoma cell lines. As shown in Fig. 2A, USP4 were efficiently knocked down by the two siRNAs in U251 and U87 cell lines. Subsequent to the UPS4 knockdown with these two siRNA sequences, glioblastoma cells were treated with TMZ. Notably, USP4 knockdown alone did not affect the cell viability, which was assessed by an MTT assay. However, when USP4 knockdown cells were treated with TMZ, the cell viability was significantly decreased compared with the scramble-transfected cells treated with TMZ, suggesting that USP4 attenuated the anti-cancer function of TMZ in the two glioblastoma cell lines (Fig. 2B and C). Furthermore, the level of cleaved PARP was also examined by western blotting in order to analyze the apoptotic status. The results demonstrated that cleaved PARP levels were elevated when cells were treated with TMZ. In addition, a marked increase in cleaved PARP was observed in cells transfected with USP4 siRNA, suggesting that USP4 inhibited the apoptosis induced by TMZ (Fig. 2D and E).

USP4 negatively regulates P53 expression. Since knockdown of USP4 promotes apoptosis, the expression of the apoptosis-associated protein P53 was then examined. The results revealed that USP4 knockdown by the two siRNA sequences led to a strong induction of P53 expression in U251 and U87 cells (Fig. 3A and B).

USP4 mediates chemoresistance via inhibiting P53 in glioblastoma. To further investigate the role of P53 in USP4-mediated chemoresistance, P53 activity was inhibited by a P53-specific inhibitor, pifithrin- $\alpha$ hydrobromide (PFT). As reported earlier, USP4 knockdown resulted in an increase in P53 expression, as well as apoptosis indicated by cleaved PARP. Following the administration of PFT, P53 protein level is slightly affected but the level of cleaved PARP reduced dramatically revealed by western blotting suggesting that USP4 facilitates chemoresistance by inhibiting P53 in both cell lines (Fig. 4A and B). Furthermore, the apoptosis mediated by USP4 knockdown was also analyzed by flow cytometry. In U251 cells, the results indicated that TMZ treatment increased the Annexin $\mathrm{V}$ and propidium iodide (PI) double-positive cells from 0.6 to $39 \%$. When USP4 knockdown cells were treated with TMZ, the Annexin V and PI double-positive cells increased to $52.5 \%$ (siRNA-1) and $45.6 \%$ (siRNA-2), suggesting that an improved chemotherapy effect may be acquired when USP4 is absent in glioblastoma. Finally, when PFT was added in 
A

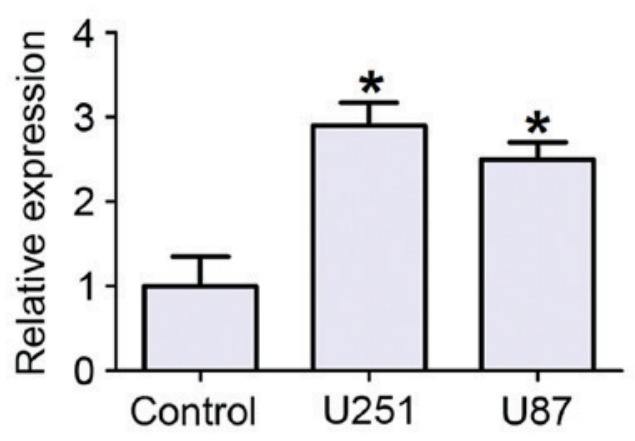

B

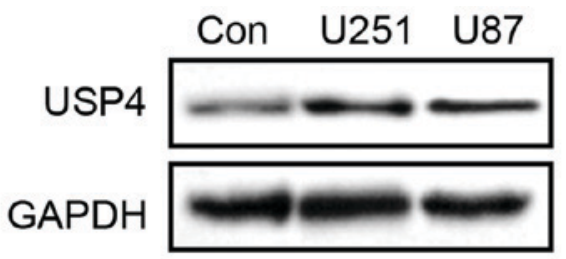

C

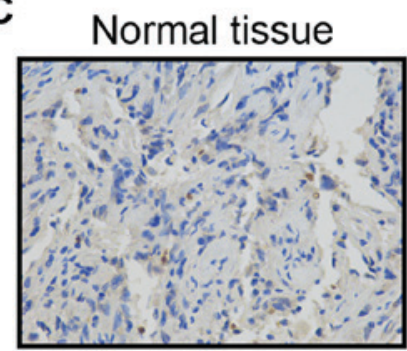

Brain carcinoma

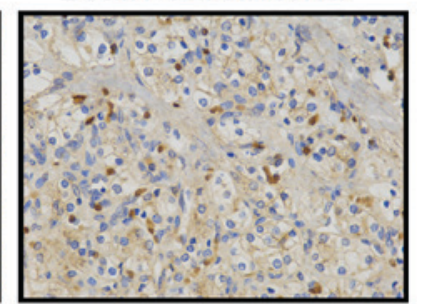

Figure 1. USP4 is highly expressed in glioblastoma. (A) Reverse transcription-quantitative polymerase chain reaction and (B) western blotting of USP4 expression levels in normal brain astrocytes (control), U251 cells and U87 cells. (C) Immunohistochemical staining for USP4 in normal brain tissue and brain carcinoma (magnification, x200). ${ }^{*} \mathrm{P}<0.05$ vs. control cells. USP4, ubiquitin-specific protease 4.
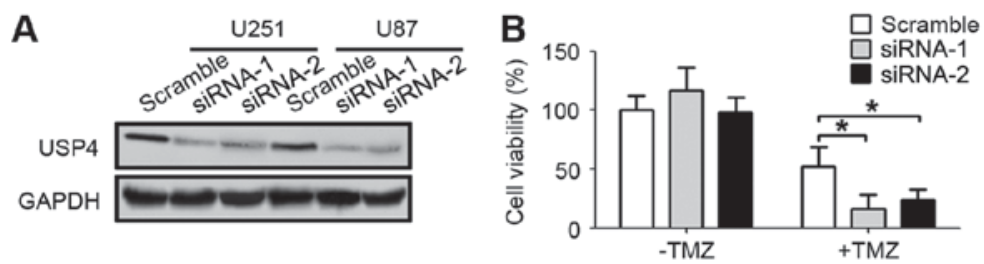
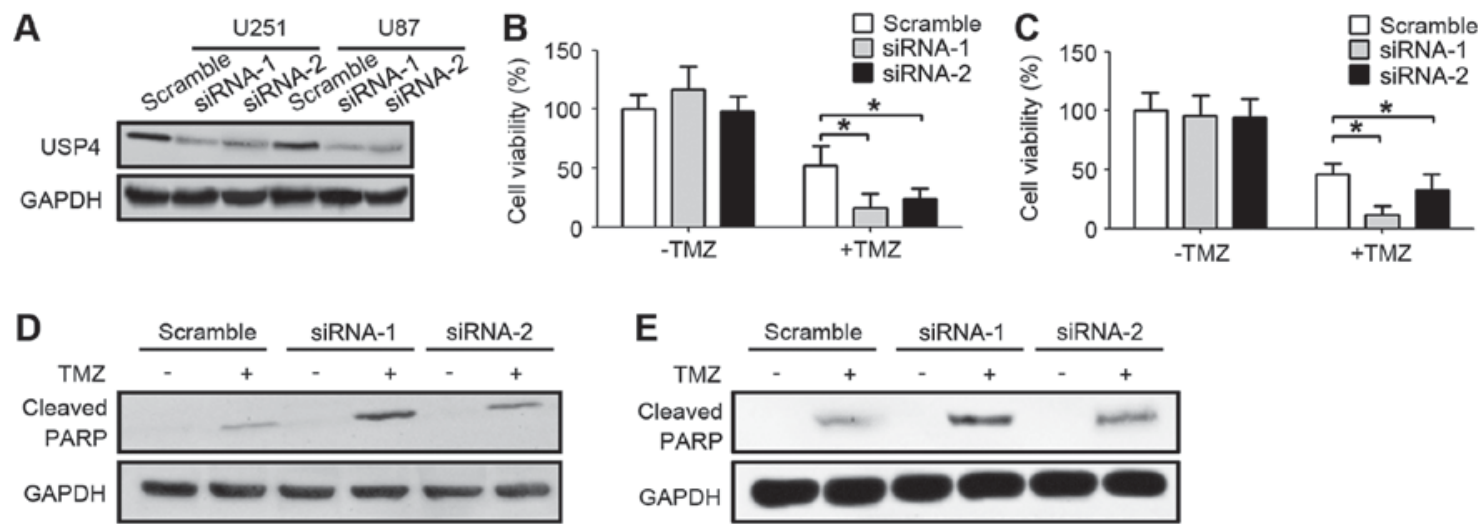

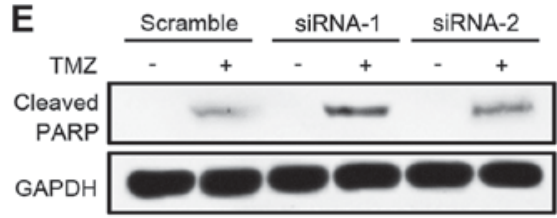

Figure 2. Downregulation of USP4 ameliorates chemoresistance of U251 and U87 cells to TMZ. (A) Western blot results showing successful knockdown of USP4 in U251 and U87 cells. (B) U251 and (C) U87 cell viability was detected by an MTT assay after $24 \mathrm{~h}$ of treatment with $100 \mu \mathrm{M}$ TMZ. Western blot analysis of cleaved PARP showing reduced chemoresistance following downregulation of USP4 in (D) U251 and (E) U87 cells. "P<0.05. USP4, ubiquitin-specific protease 4; TMZ, temozolomide; PARP, poly(ADP-ribose) polymerase.

A

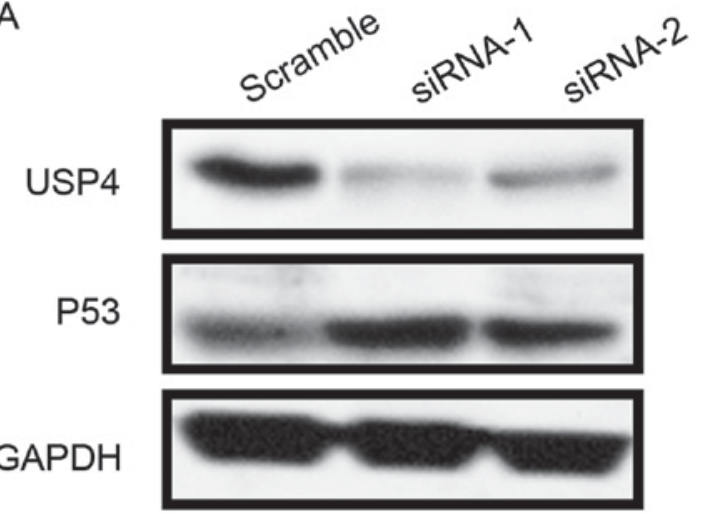

B

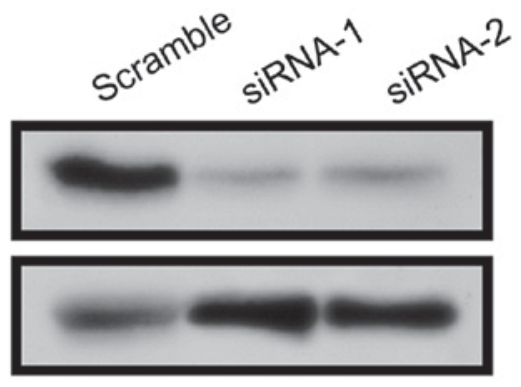

GAPDH

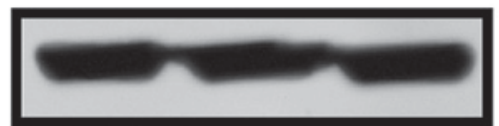

Figure 3. USP4 negatively regulates P53 activity. (A) U251 and (B) U87 cells were transfected with scramble or USP4 small interfering RNAs, and then USP4 and P53 levels were detected by western blotting at $48 \mathrm{~h}$ post-transfection. USP4, ubiquitin-specific protease 4.

TMZ-treated USP4 knockdown cells, the percentage of apoptotic cells was decreased to $42.0 \%$ (siRNA-1) and $35.4 \%$ (siRNA-2), suggesting that USP4-mediated chemoresistance is dependent on P53 activity (Fig. 4C). Similarly, in U87 cells, USP4 knockdown facilitated the drug effect of TMZ, while P53 inhibitor reversed this effect (Fig. 4D). 

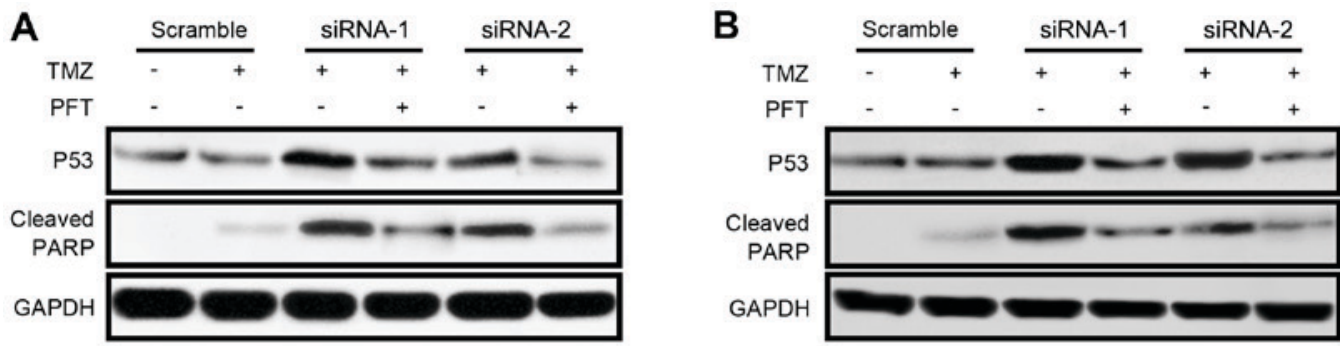

C

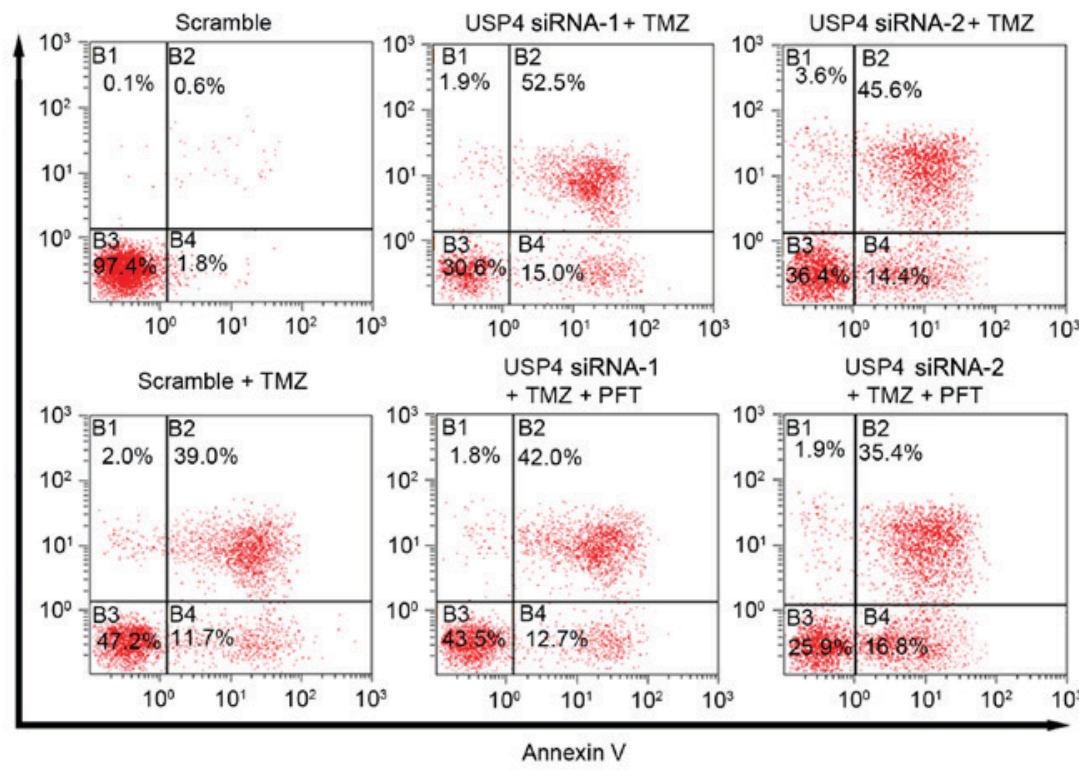

D

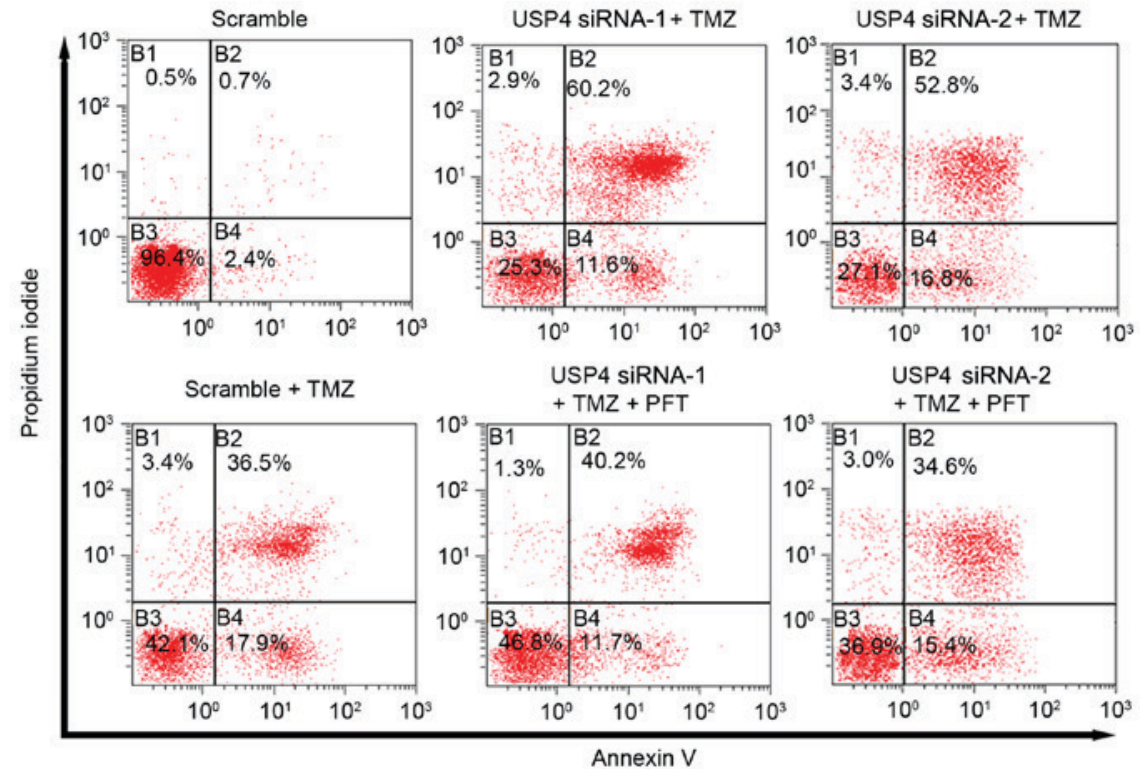

Figure 4. Inhibiting P53 activity in USP4 knockdown cells resumes chemoresistance to TMF. (A) U251 and (B) U87 cells transfected with scramble or USP4 small interfering RNAs were treated with $100 \mu \mathrm{M} \mathrm{TMZ}$ with or without $30 \mu \mathrm{M}$ PFT for $24 \mathrm{~h}$, and then P53 and cleaved PARP levels were detected by western blotting. (C) U251 and (D) U87 cells were examined by flow cytometry analysis, and the results indicated that PFT treatment resumed the chemoresistance of USP4 knockdown cells to TMZ. USP4, ubiquitin-specific protease 4; TMZ, temozolomide; PFT, pifithirin- $\alpha$ hydrobromide; PARP, poly(ADP-ribose) polymerase.

\section{Discussion}

Glioblastoma is the most common primary malignancy of the brain. In spite of decades of research on this disease, little progress has been made to improve the survival rate of patients. Resistance to anticancer drugs is a problem in numerous types of cancer, particularly in glioblastoma (6). Combining radiation therapy with TMZ is currently the first-line therapy for glioblastoma. However, the efficiency of TMZ remains limited owing to inherent and acquired resistance of glial tumor cells. It is, thus, urgent to determine the underlying mechanism that genetic alterations of patients facilitate drug resistances. 
The present study findings revealed that USP4 is significantly upregulated in glioblastoma tissues, as well as in glioblastoma cell lines. It was observed that, when USP4 was knocked down, glioblastoma cells became more sensitive to TMZ treatment, suggesting the pro-cancer role of USP4. Furthermore, the results demonstrated that P53 was increased in USP4 knockdown U251 and U87 cells. Taken together, the results of the present study suggested that USP4 mediated chemoresistance through inhibiting apoptosis in a P53-dependent manner.

Due to the critical role of $\mathrm{p} 53$ in a variety of tumors, targeting p53 and its altered signaling pathways is of great importance in order to gain a better prognosis, particularly in malignancies such as glioblastoma. Thus, it is critical to understand the genetic background that affects the normal function of P53 signaling. The findings of the current study suggested a de novo role of USP4 in chemoresistance via P53 signaling, providing new evidence for future drug discovery and clinical treatment of glioblastoma. However, despite extensive studies, the data regarding a link between altered p53 and efficacy of chemotherapy in patients with glioblastoma remain controversial (37). Certain studies have argued that the p53 status correlates with a favorable response of patients to therapy $(38,39)$, while other studies revealed no such correlation $(40,41)$. Thus, it is of great use to further explore the role of P53 in chemoresistance and to develop therapies that simultaneously target P53 and USP4 in order to improve the outcome of chemotherapy.

In conclusion, the results reported in the current study suggested a pro-cancer role of USP4 in glioblastoma by facilitating chemoresistance. Thus, to acquire better benefits for glioblastoma patients with elevated USP4 expression, it is useful to develop drugs that antagonize USP4. In addition, USP4 function in various types of cancer requires further investigations. Experiments in breast cancer indicated that USP4 was downregulated in breast cancer tissues, while USP4 overexpression led to inhibition of breast cancer cell proliferation (42). Furthermore, how USP4 affects P53 signaling and whether USP4 mediates TMZ resistance through other types of signaling remain unclear. However, USP4 appears to be a potential target for future drug discovery for glioblastoma.

\section{Acknowledgements}

The authors thank Dr Gang Wu (Department of Neurology, Xijing Hospital of the Fourth Military Medical University) for providing glioblastoma tissue samples obtained from patients.

\section{Funding}

This study was supported by grants from the National Natural Science Foundation of China (nos. 81402544 and 31370834).

\section{Availability of data and materials}

All data generated or analyzed during the present study are included in this published article.

\section{Authors' contributions}

Cell culture and associated treatments were performed by NQ and FH. NQ and YG performed qPCR assays, FH and
LL performed western blotting. NQ and YG performed flow cytometry. LW performed cell viability analysis. GZ performed the histological examination of the brain tissue. NQ, FH and LL wrote the manuscript. YD and JZ designed this study and contributed to manuscript revision. All authors read and approved the final manuscript.

\section{Ethics approval and consent to participate}

The present study was approved by the Ethics Committee of the Fourth Military Medical University (Xi'an, China). Written informed consent was obtained from all patients.

\section{Patient consent for publication}

Written informed consent was obtained from all the patients.

\section{Competing interests}

The authors declare that they have no competing interests.

\section{References}

1. Wen PY and Kesari S: Malignant gliomas in adults. N Engl J Med 359: 492-507, 2008.

2. Scott CB, Scarantino C, Urtasun R, Movsas B, Jones CU, Simpson JR, Fischbach AJ and Curran WJ Jr: Validation and predictive power of Radiation Therapy Oncology Group (RTOG) recursive partitioning analysis classes for malignant glioma patients: A report using RTOG 90-06. Int J Radiat Oncol Biol Phys 40: 51-55, 1998.

3. Jackson CM, Lim M and Drake CG: Immunotherapy for brain cancer: Recent progress and future promise. Clin Cancer Res 20: 3651-3659, 2014

4. Wilson TA, Karajannis MA and Harter DH: Glioblastoma multiforme: State of the art and future therapeutics. Surg Neurol Int 5: 64, 2014

5. Oike T, Suzuki Y, Sugawara K, Shirai K, Noda SE, Tamaki T, Nagaishi M, Yokoo H, Nakazato Y and Nakano T: Radiotherapy plus concomitant adjuvant temozolomide for glioblastoma: Japanese mono-institutional results. PLoS One 8: e78943, 2013.

6. Stupp R, Mason WP, van den Bent MJ, Weller M, Fisher B, Taphoorn MJ, Belanger K, Brandes AA, Marosi C, Bogdahn U, et al: Radiotherapy plus concomitant and adjuvant temozolomide for glioblastoma. N Engl J Med 352: 987-996, 2005.

7. Stupp R, Hegi ME, Mason WP, van den Bent MJ, Taphoorn MJ, Janzer RC, Ludwin SK, Allgeier A, Fisher B, Belanger K, et al: Effects of radiotherapy with concomitant and adjuvant temozolomide versus radiotherapy alone on survival in glioblastoma in a randomised phase III study: 5-year analysis of the EORTC-NCIC trial. Lancet Oncol 10: 459-466, 2009.

8. Messaoudi K, Clavreul A and Lagarce F: Toward an effective strategy in glioblastoma treatment. Part I: Resistance mechanisms and strategies to overcome resistance of glioblastoma to temozolomide. Drug Discov Today 20: 899-905, 2015.

9. Messaoudi K, Clavreul A and Lagarce F: Toward an effective strategy in glioblastoma treatment. Part II: RNA interference as a promising way to sensitize glioblastomas to temozolomide. Drug Discov Today 20: 772-779, 2015.

10. Esteller M, Garcia-Foncillas J, Andion E, Goodman SN, Hidalgo OF, Vanaclocha V, Baylin SB and Herman JG: Inactivation of the DNA-repair gene MGMT and the clinical response of gliomas to alkylating agents. N Engl J Med 343: 1350-1354, 2000.

11. Huang PH, Xu AM and White FM: Oncogenic EGFR signaling networks in glioma. Sci Signal 2: re6, 2009.

12. Paz A, Haklai R, Elad-Sfadia G, Ballan E and Kloog Y: Galectin-1 binds oncogenic $\mathrm{H}$-Ras to mediate Ras membrane anchorage and cell transformation. Oncogene 20: 7486-7493, 2001.

13. Burton EC, Lamborn KR, Forsyth P, Scott J, O'Campo J, Uyehara-Lock J, Prados M, Berger M, Passe S, Uhm J, et al: Aberrant p53, mdm2, and proliferation differ in glioblastomas from long-term compared with typical survivors. Clin Cancer Res 8: 180-187, 2002. 
14. Wang AL, Liu ZX, Li G and Zhang LW: Expression and significance of P53 protein and MDM-2 protein in human gliomas. Chin Med J (Engl) 124: 2530-2533, 2011.

15. Kato H, Kato S, Kumabe T, Sonoda Y, Yoshimoto T, Kato S, Han SY, Suzuki T, Shibata H, Kanamaru R and Ishioka C: Functional evaluation of p53 and PTEN gene mutations in gliomas. Clin Cancer Res 6: 3937-3943, 2000.

16. Babashah S and Soleimani M: The oncogenic and tumour suppressive roles of microRNAs in cancer and apoptosis. Eur J Cancer 47: 1127-1137, 2011.

17. Mizoguchi M, Guan Y, Yoshimoto K, Hata N, Amano T, Nakamizo A and Sasaki T: Clinical implications of microRNAs in human glioblastoma. Front Oncol 3: 19, 2013.

18. Vogelstein B, Lane D and Levine AJ: Surfing the p53 network. Nature 408: 307-310, 2000

19. Toledo F and Wahl GM: Regulating the p53 pathway: In vitro hypotheses, in vivo veritas. Nat Rev Cancer 6: 909-923, 2006.

20. Robles AI and Harris CC: Clinical outcomes and correlates of TP53 mutations and cancer. Cold Spring Harb Perspect Biol 2: a001016, 2010

21. Hermisson M, Klumpp A, Wick W, Wischhusen J, Nagel G, Roos W, Kaina B and Weller M: O6-methylguanine DNA methyltransferase and p53 status predict temozolomide sensitivity in human malignant glioma cells. J Neurochem 96: 766-776, 2006.

22. Wang X, Chen JX, Liu YH, You C and Mao Q: Mutant TP53 enhances the resistance of glioblastoma cells to temozolomide by up-regulating $\mathrm{O}(6)$-methylguanine DNA-methyltransferase. Neurol Sci 34: 1421-1428, 2013.

23. Nie E, Jin X, Wu W, Yu T, Zhou X, Zhi T, Shi Z, Zhang J, Liu N and You Y: BACH1 promotes temozolomide resistance in glioblastoma through antagonizing the function of p53. Sci Rep 6: 39743, 2016.

24. Nijman SM, Luna-Vargas MP, Velds A, Brummelkamp TR, Dirac AM, Sixma TK and Bernards R: A genomic and functional inventory of deubiquitinating enzymes. Cell 123: 773-786, 2005.

25. Fang X, Zhou W, Wu Q, Huang Z, Shi Y, Yang K, Chen C, Xie Q, Mack SC, Wang X, et al: Deubiquitinase USP13 maintains glioblastoma stem cells by antagonizing FBXL14-mediated Myc ubiquitination. J Exp Med 214: 245-267, 2017.

26. Li M, Chen D, Shiloh A, Luo J, Nikolaev AY, Qin J and Gu W: Deubiquitination of p53 by HAUSP is an important pathway for p53 stabilization. Nature 416: 648-653, 2002.

27. Wang CL, Wang JY, Liu ZY, Ma XM, Wang XW, Jin H, Zhang XP, Fu D, Hou LJ and Lu YC: Ubiquitin-specific protease 2a stabilizes MDM4 and facilitates the p53-mediated intrinsic apoptotic pathway in glioblastoma. Carcinogenesis 35: 1500-1509, 2014

28. Zhang X, Berger FG, Yang J and Lu X: USP4 inhibits p53 through deubiquitinating and stabilizing ARF-BP1. EMBO J 30 $2177-2189,2011$

29. Li Z, Hao Q, Luo J, Xiong J, Zhang S, Wang T, Bai L, Wang W, Chen M, Wang W, et al: USP4 inhibits p53 and NF- $\mathrm{B}$ through deubiquitinating and stabilizing HDAC2. Oncogene 35: 2902-2912, 2016.

30. Zhang L, Zhou F, Drabsch Y, Gao R, Snaar-Jagalska BE, Mickanin C, Huang H, Sheppard KA, Porter JA, Lu CX and ten Dijke P: USP4 is regulated by AKT phosphorylation and directly deubiquitylates TGF- $\beta$ type I receptor. Nat Cell Biol 14: 717-726, 2012.
31. Hwang SJ, Lee HW, Kim HR, Lee H, Shin CH, Yun SI, Lee DH, Kim DH, Kim KK, Joo KM and Kim HH: Ubiquitin-specific protease 4 controls metastatic potential through $\beta$-catenin stabilization in brain metastatic lung adenocarcinoma. Sci Rep 6: 21596, 2016.

32. Liu C, Liu C, Liu H, Gong L, Tao T, Shen Y, Zhu S and Shen A: Increased expression of ubiquitin-specific protease 4 participates in neuronal apoptosis after intracerebral hemorrhage in adult rats. Cell Mol Neurobiol 37: 427-435, 2017.

33. Fraser M, Leung BM, Yan X, Dan HC, Cheng JQ and Tsang BK: p53 is a determinant of X-linked inhibitor of apoptosis protein/Akt-mediated chemoresistance in human ovarian cancer cells. Cancer Res 63: 7081-7088, 2003.

34. Louis DN, Perry A, Reifenberger G, von Deimling A, Figarella-Branger D, Cavenee WK, Ohgaki H, Wiestler OD, Kleihues P and Ellison DW: The 2016 world health organization classification of tumors of the central nervous system: A summary. Acta Neuropathol 131: 803-820, 2016.

35. Livak KJ and Schmittgen TD: Analysis of relative gene expression data using real-time quantitative PCR and the 2(-Delta Delta $\mathrm{C}(\mathrm{T}))$ method. Methods 25: 402-408, 2001.

36. Romanowska M, Evans A, Kellock D, Bray SE, McLean K, Donandt S and Foerster J: Wnt5a exhibits layer-specific expression in adult skin, is upregulated in psoriasis, and synergizes with type 1 interferon. PLoS One 4: e5354, 2009.

37. Masui K, Cloughesy TF and Mischel PS: Review: Molecular pathology in adult high-grade gliomas: From molecular diagnostics to target therapies. Neuropathol Appl Neurobiol 38: 271-291, 2012.

38. Schiebe M, Ohneseit P, Hoffmann W, Meyermann R, Rodemann HP and Bamberg M: Analysis of mdm2 and p53 gene alterations in glioblastomas and its correlation with clinical factors. J Neurooncol 49: 197-203, 2000.

39. Birner P, Piribauer M, Fischer I, Gatterbauer B, Marosi C, Ungersböck K, Rössler K, Budka H and Hainfellner JA: Prognostic relevance of $\mathrm{p} 53$ protein expression in glioblastoma. Oncol Rep 9: 703-707, 2002.

40. Kraus JA, Wenghoefer M, Glesmann N, Mohr S, Beck M, Schmidt MC, Schröder R, Berweiler U, Roggendorf W, Diete $\mathrm{S}$, et al: TP53 gene mutations, nuclear $\mathrm{p} 53$ accumulation, expression of Waf/p21, Bcl-2, and CD95 (APO-1/Fas) proteins are not prognostic factors in de novo glioblastoma multiforme. J Neurooncol 52: 263-272, 2001.

41. Rich JN, Hans C, Jones B, Iversen ES, McLendon RE, Rasheed BK, Dobra A, Dressman HK, Bigner DD, Nevins JR and West M: Gene expression profiling and genetic markers in glioblastoma survival. Cancer Res 65: 4051-4058, 2005.

42. Li Y, Jiang D, Zhang Q, Liu X and Cai Z: Ubiquitin-specific protease 4 inhibits breast cancer cell growth through the upregulation of PDCD4. Int J Mol Med 38: 803-811, 2016.

(i) (9) This work is licensed under a Creative Commons Attribution-NonCommercial-NoDerivatives 4.0 International (CC BY-NC-ND 4.0) License. 